\title{
P 023 ADAPTATION, FACE AND CONTENT VALIDATION OF A PALLIATIVE CARE NEEDS ASSESSMENT TOOL FOR PEOPLE WITH INTERSTITIAL LUNG DISEASE
}

C Reigada, ${ }^{1}$ S Bajwah, ${ }^{2}$ J Ross, ${ }^{3}$ J Boland, ${ }^{1}$ A Wells, ${ }^{3}$ J Yorke, ${ }^{4}$ G Grande, ${ }^{4}$ S Hart, ${ }^{5}$ D Currow, ${ }^{6}$ T Papadopoulos, ${ }^{7}$ U Macleod, ${ }^{1}$ M Johnson ${ }^{1}$. ${ }^{1}$ Hull York Medical School, University of Hull; ${ }^{2}$ King's College Hospital NHS Foundation Trust \& Cicely Saunders Institute, Kings College, London; ${ }^{3}$ Royal \& Harefield NHS Foundation Trust; ${ }^{4}$ University of Manchester; ${ }^{5}$ Hull and East of Yorkshire Hospitals NHS Trust; ${ }^{6}$ Discipline, Palliative and Supportive Services, Flinders University, Australia; ${ }^{7}$ School of Business, Management, and Economics, University of Sussex

\subsection{6/bmjspcare-2014-000838.26}

Introduction Interstitial lung disease (ILD) is a non-malignant progressive disease which causes breathlessness, cough, fatigue, psycho-social, financial and spiritual distress; difficult for patients and their family carers. The Needs Assessment Tool: Progressive Disease (NAT:PD-C) was developed as an "aidememoire" to help clinicians identify a range of palliative care needs in people with cancer, and their carers, in daily practice. We have adapted this tool for people with ILD.

Aim(s) and method(s) Our aim was to adapt, and then test face and content validity of the adapted version.

The NAT:PD-C was adapted for people with ILD using data from a systematic review of the literature and previously completed qualitative interviews. Focus groups were held (patients $[N=7]$, carers $[N=3]$, clinicians $[N=8]$ followed by an Expert Consensus Group to discuss face and content validity of the NAT:PD-ILD. The Expert Group also discussed issues regarding implementation in practice. The 


\section{Abstracts}

discussion was audio and video-recorded, and cognitive mapping was used. Data was analysed following the principles of inductive theory building. Further validation and reliability testing is ongoing. We present the preliminary results of the face/content validation.

Results All sections ("red flags" for high risk patients; priority referral; patient wellbeing; ability of carer to provide care; carer wellbeing) of the tool were felt to be important. Some symptoms (voice, mucous and mobility) were added by patients. Clinicians identified training needs about "Spiritual or existential concerns". Carers highlighted the need for support for themselves.

Conclusion(s) The NAT:PD-ILD appears to have face and content validity and the content and structure reflected the experience of all groups.

*This research was funding by Marie Curie Care Cancer Research UK. 